\title{
Optical detection of aqueous phase analytes via host-guest interactions on a lipid membrane surface
}

\author{
Darryl Y. Sasaki* and Tina A. Waggoner \\ Sandia National Laboratories, MS 1407, Albuquerque, NM 87185
}

\begin{abstract}
The organization and assembly of molecules in cellular membranes is orchestrated through the recognition and binding of specific chemical signals. A simplified version of the cellular membrane system has been developed using a synthetically prepared membrane receptor incorporated into a biologically derived lipid bilayer. Through an interplay of electrostatic and van der Waals interactions, aggregation or dispersion of molecular components could be executed on command using a specific chemical signal. A pyrene fluorophore was used as an optical probe to monitor the aggregational state of the membrane receptors in the bilayer matrix. The pyrene excimer emission to monomer emission (E/M) intensity ratio gave a relative assessment of the local concentration of receptors in the membrane. Bilayers were prepared with receptors selective for the divalent metal ions of copper, mercury, and lead. Addition of the metal ions produced a rapid dispersion of aggregated receptor components at nano- to micro-molar concentrations. The process was reversible by sequestering the metal ions with EDTA. Receptors for proteins and polyhistidine were also prepared and incorporated into phosphatidylcholine lipid bilayers. In this case, the guest molecules bound to the membrane through multiple points of interaction causing aggregation of initially dispersed receptor molecules. The rapid, selective, and sensitive fluorescence optical response of these lipid assemblies make them attractive in sensor applications for aqueous phase metal ions and polypeptides.
\end{abstract}

Keywords: molecular reorganization, membrane receptor, lipid bilayer, metal ion sensor, protein binding, fluorescence, pyrene

\section{INTRODUCTION}

Molecular organization in cellular membranes is a precisely orchestrated dance of various membrane components responding to chemical and physical forces. Membrane receptors respond to the host-guest binding interaction of signaling molecules captured selectively from the extracellular or intracellular milieu. ${ }^{1}$ The receptors can organize into clusters, such as integrin receptors, ${ }^{2}$ or into complex assemblies, such as those associated with T-cell receptors. ${ }^{3}$ This perfect organization, initiated by the molecular recognition event of a binding ligand, is driven by an array of interactions. The exact nature of each interaction and their effect on the order and structure of the molecular organization is an area that is largely unexplored. Investigations that can isolate specific interactions and their effect on molecular assemblies will provide a framework of understanding into molecular organization in dynamic self-assembled systems.

The recognition of specific molecular ligands on cellular membrane surfaces typically initiates an assembly of receptor components into an aggregate that subsequently activates intercellular proteins and the signal cascade process. Release of the ligand then disassembles the receptor components shutting down the cellular signal process. Mimicry of such reversible organization of components in lipid membranes may be achieved using simplified receptors to analytes that act as "triggers" to effect the process. Synthetic receptors, prepared and incorporated into biomimetic lipid bilayers, can utilize physical and chemical interactions to assemble in different configurations. Binding of chemical analytes could in turn effect physical (e.g., phase transition temperature $T_{g}$, ionic charge) or chemical (e.g., hydrogen bonding, acid-base coordination) changes in the receptors and subsequent reorganization of the matrix. Figure 1 illustrates an example of a simple molecular reorganization of a two component system that switches between an aggregated to dispersed assembly. By using a reversible host-guest interaction with an appropriate design of the bilayer matrix we can begin to investigate controlled molecular organization via chemical signals.

Herein, we will describe the design, preparation, and characterization of a simple two component lipid bilayer assembly that switches reversibly between an aggregated to dispersed molecular organization via a metal ion chemical signal. One of the

Correspondence: e-mail: dysasak@sandia.gov: Telephone: (505)845-0824; FAX: (505)844-9624. 


\section{DISCLAIMER}

This report was prepared as an account of work sponsored by an agency of the United States Government. Neither the United States Government nor any agency thereof, nor any of their employees, make any warranty, express or implied, or assumes any legal liability or responsibility for the accuracy, completeness, or usefulness of any information, apparatus, product, or process disclosed, or represents that its use would not infringe privately owned rights. Reference herein to any specific commercial product, process, or service by trade name, trademark, manufacturer, or otherwise does not necessarily constitute or imply its endorsement, recommendation, or favoring by the United States Government or any agency thereof. The views and opinions of authors expressed herein do not necessarily state or reflect those of the United States Government or any agency thereof. 


\section{DISCLAIMER}

Portions of this document may be illegible in electronic image products. Images are produced from the best available original document. 
components is a synthetic lipid with a metal ion receptor as a headgroup and a pyrene fluorophore on the hydrophobic tail. Sonication in aqueous solution of this receptor lipid with a biologically derived phosphatidylcholine lipid generates functionalized bilayer assemblies. Appropriate selection of the two lipid components gave rise to membranes with receptors aggregated in pools as a byproduct of phase separation. Binding of metal ion creates a positive charge at the headgroup position leading to electrostatic repulsion and subsequent dispersion of the aggregated receptors. In other experiments, we also show that aggregation of membrane receptors could be achieved using a multiple-point binding interaction between polypeptides and a functionalized bilayer. Fluorescence spectral data from the pyrene label served as an optical monitor for the reorganization processes of the receptor lipids. Results of tailoring the chemical specificity to selected metal ions, proteins, and polypeptides will also be discussed.

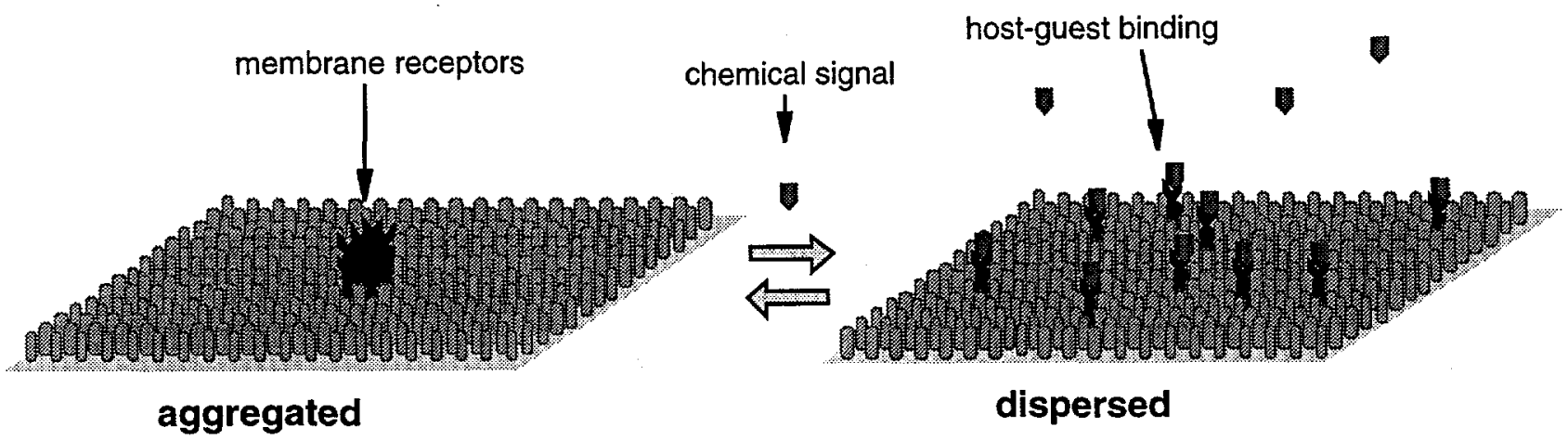

Figure 1. Molecular reorganization in two-dimensional molecular assemblies controlled via chemical signaling.

\section{PREPARATION OF SYNTHETIC RECEPTORS AND LIPID BILAYERS}

Functionalized lipid bilayers were prepared by dispersing a mixture of two lipid components in buffered water. One component is a phosphatidylcholine lipid that serves as the matrix of the bilayers. The other lipid is a totally synthetic molecule functionalized with a metal ion receptor at the headgroup position and pyrene fluorophore tag on the hydrophobic tail. In this section we will briefly describe the synthetic preparation of the functionalized lipids and technique used to generate the functionalized bilayer structures. Characterization of the bilayers was performed with transmission electron microscopy (TEM) and fluorescence spectroscopy.

\subsection{Design and Synthesis of Metal Ion Receptor Lipids}

The metal ion receptor lipids were designed in the structural image of a biological glycerol-based lipid, such as the phosphatidylcholines. Figure 2 shows the molecular structure of the receptor lipid and the different ion chelating headgroups used to prepare PSIDA, PSDSDA, PS18C6, and the non-metal-chelating lipid PSOH. In the central part of the molecule is the glycerol backbone, which connects two hydrophobic long alkane chains, at the $\mathrm{C} 1$ and $\mathrm{C} 2$ positions of the glycerol, to a hydrophilic headgroup at the C3 position. To enhance the chemical stability of the lipid the typical ester linkages of biological lipids were replaced with ethers. A pyrene fluorescent probe was tagged onto the $C 2$ alkane chain to be used as an optical monitor of the lipid aggregational state in the bilayer. The hydrophilic moiety at the C3 position is a triethyleneglycol spacer unit functionalized at the distal end with the metal ion receptor. The triethyleneglycol spacer is used to extend the receptor headgroup into the bulk solution away from the membrane surface.

The synthetic preparation of the PS lipids involves multiple synthetic steps, which are described in detail elsewhere. ${ }^{4,5,6}$ Each of the metal ion receptor lipids is a derivative of the hydroxy terminated PSOH. Conversion to the different headgroups required an activation of the hydroxy group followed by a substitution reaction with the appropriate nucleophilic analog of the metal receptor. For example, PS18C6 was prepared by first substituting the hydroxy group on PSOH with a bromine, then reacting the bromide compound with (18-crown-6)methanol under basic conditions. The PS lipid products were isolated by flash column chromatography as slightly yellow viscous oils or waxy solids.

All PS lipids formed stable Langmuir monolayers at the air-water interface indicating good amphiphilic properties. The ability to form stable Langmuir monolayers reveals a hydrophilic headgroup and a tendency of these linear molecules to 
orient in a direction normal to the aqueous solution. ${ }^{7,8}$ Inadequate hydration of the headgroup could lead to poor receptor presentation to the bulk solution or even lack of incorporation into lipid bilayers. The pressure-area isotherms of each PS lipid are shown in Figure 3. The isotherms for the PSOH and PSIDA lipids gave steep slopes near their molecular crosssections $\left(60 \AA^{2}\right)$ suggesting headgroups with strong hydrophilic character. The shallower slopes for PSDSDA and PS18C6 indicate headgroups with less hydrophilic property. This difference in hydrophilic property of the PS lipids and their possible effects on the resultant structures of the bilayers incorporating these lipids will be discussed in the next section.
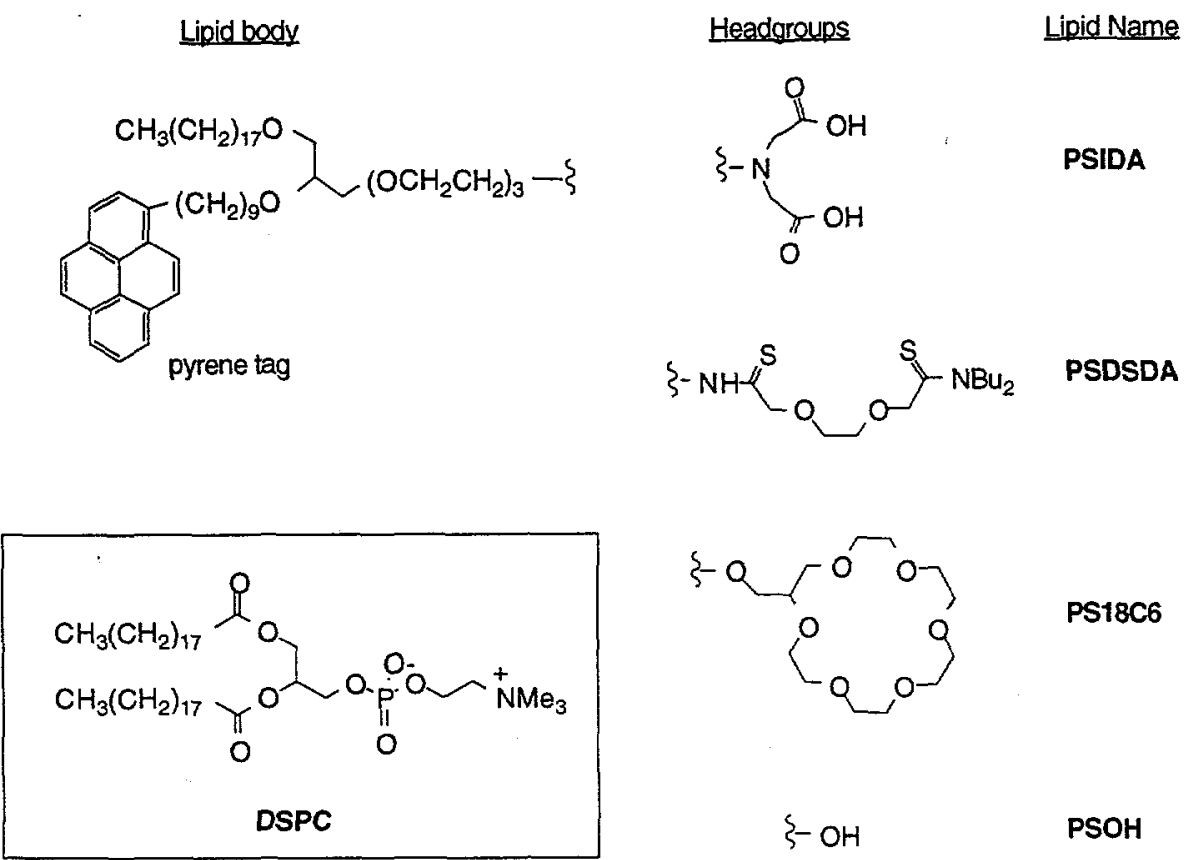

Figure 2. Molecular structures of synthetically prepared metal ion receptor lipids PSIDA. PSDSDA. and PS18C6. along with their precursor PSOH. The matrix lipid DSPC is also shown.

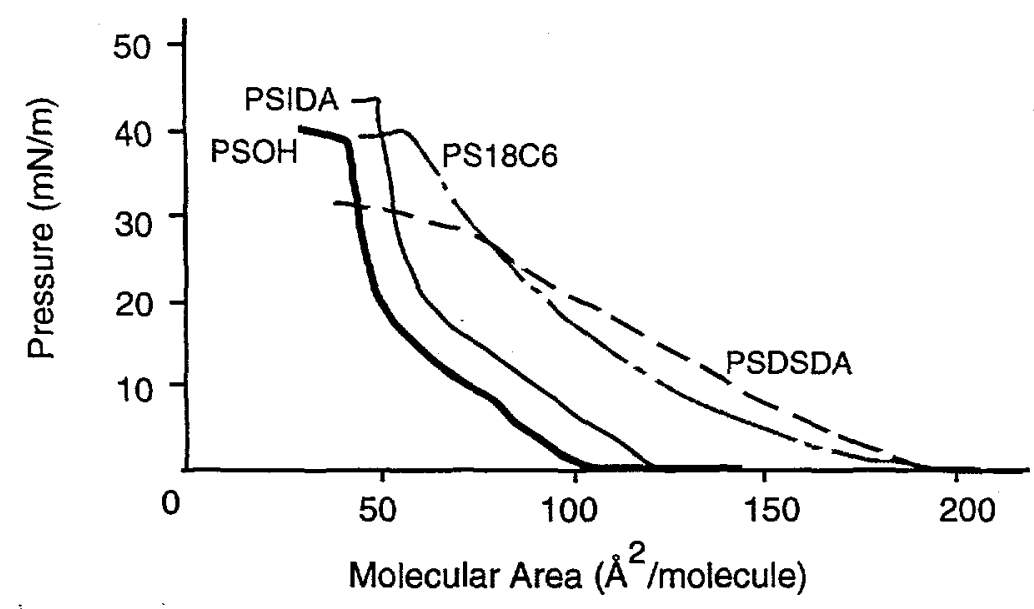

Figure 3. Pressure-area isotherms of PSOH, PSIDA, PSDSDA, and PS18C6, performed on a pure water subphase at $20^{\circ} \mathrm{C}$.

\subsection{Formation of Lipid Bilayers}

The two component bilayer structures were formed using standard techniques for producing small unilamellar vesicles, or SUV's.' An SUV is a single, continuous bilayer structure constituting a spherical or oblate shape. The SUV is ca. $300 \AA$ in 
diameter, the minimum size of an unsupported lipid bilayer structure. Although lipid membranes could be formed into a variety of different structures, the SUV provides the best conditions for the current studies. Of the unsupported bilayer structures, the SUV provides maximum surface exposure to the bulk solution. Since the receptors must be in contact with the bulk solution to capture the chemical signal, any receptors entrapped within a vesicle, such as with multilamellar vesicles (MLV's), ${ }^{9}$ are masked to the introduction of the signal. These masked receptors will then diminish the optical response. For any vesicle structure the inner leaflet of the bilayer will also be unexposed to the bulk solution. However, the ratio of the amount of lipids in the inner to outer leaflet is minimized with the SUV, considering geometrical constraints. ${ }^{10}$ Thus, of the possible bilayer membrane structures, maximum receptor exposure and membrane response to chemical signals should be achieved using the SUV.

Preparation of the SUV was as follows: A phosphatidylcholine lipid (i.e., distearylphosphatidylcholine (DSPC) or 1-stearyl2-oleoyl-sn-glycerophosphatidylcholine (SOPC)) and a PS lipid were dissolved in chloroform in a 95:5 molar ratio at a total lipid concentration of $10 \mathrm{mM}$. The solution was evaporated to a thin film on the walls of a glass centrifuge tube using a rotary evaporator. Residual solvent was removed from the film by further drying overnight under high vacuum. The film was then hydrated in $3 \mathrm{~mL}$ of MOPS buffer solution ( $20 \mathrm{mM}$ 4-morpholinopropanesulfonic acid, $100 \mathrm{mM} \mathrm{NaCl}, \mathrm{pH} 7.4)$ at $60-65{ }^{\circ} \mathrm{C}$ with vortex stirring. The completely suspended film was first degassed with $\mathrm{N}_{2}$ gas for several minutes, then sonicated with a 3-mm probe tip at $25 \mathrm{~W}$ power. Sonication was performed in 4 minutes cycles with one minute resting in between for a total of 20 minutes. The translucent solution was centrifuged for 30 minutes at $16,000 \mathrm{~g}$ to remove metal bits from the sonication probe and large bilayer aggregates. The clear supernatant was filtered through a 0.2 micron filter and the final solution diluted with MOPS buffer to a desired lipid concentration. Fluorescence experiments were generally performed with total lipid concentrations of between 0.5 and $5.0 \mu \mathrm{M}$.

Total lipid incorporation into the bilayer structures was typically around $50 \%$ of the initial lipid concentration, as determined by phosphate analysis. ${ }^{9}$ Transmission electron micrographs (TEM) of the $5 \%$ PSIDA/DSPC and 5\% PSDSDA/DSPC bilayer structures are shown in Figure 4A and $4 \mathrm{~B}$, respectively. The PSIDA containing bilayers formed spherical structures (liposomes) with an average diameter of ca. $300 \AA$. In comparison, bilayers containing the PSDSDA lipid gave rise to disklike bilayer structures, also ca. $300 \AA$ in length. Larger aggregates of these disks are also present in the TEM. Considering the only difference between the two bilayer structures of Figure 4A and 4B is the headgroup of a lipid present at $5 \%$ in the membrane, there is a remarkably large difference in aggregate structure. Of the possible causes of the differences in the resultant bilayer structures, hydrophilicity of the metal ion receptor may be a factor. As was observed with the monolayer pressure-area studies, PSIDA has better orientation to the air-water interface than PSDSDA suggesting a more hydrophilic headgroup. Further investigations into this effect of the headgroup chemistry of a "dopant" lipid dictating the structure of bilayer assemblies are being pursued.
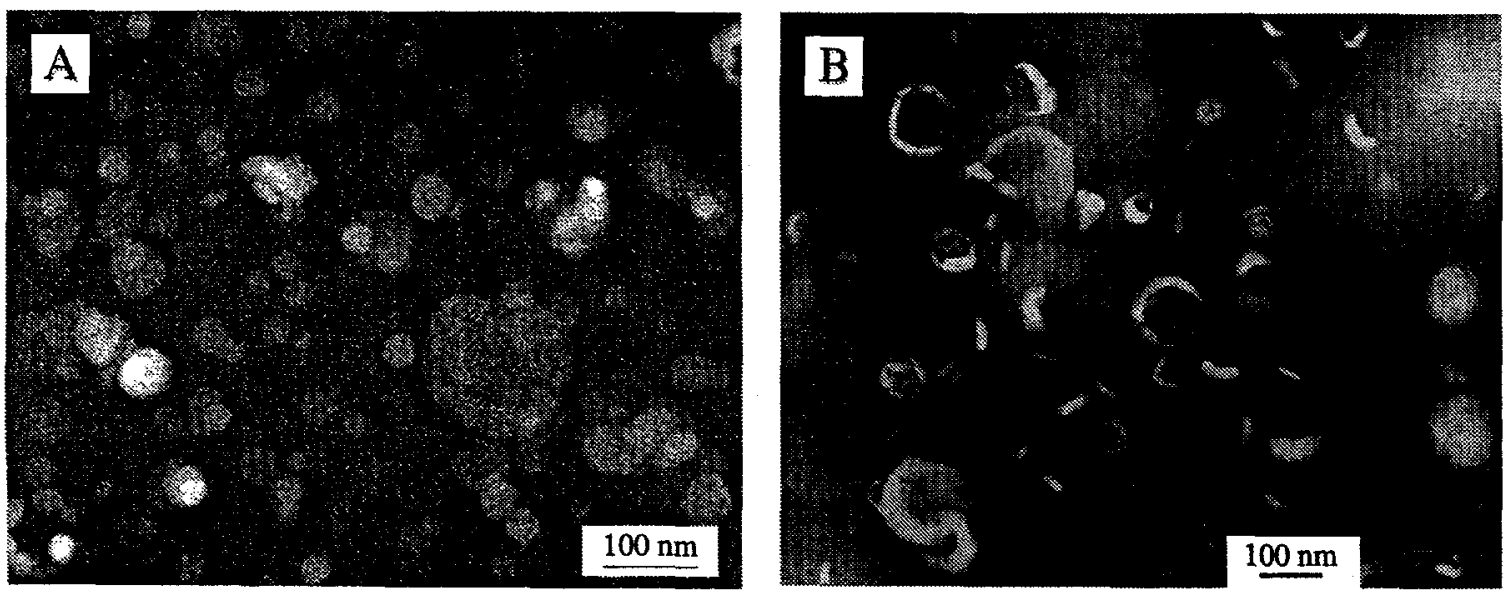

Figure 4. TEM micrographs of (A) 5\% PSIDA/DSPC and (B) 5\% PSDSDA/DSPC lipid bilayers deposited from aqueous buffer solution and stained with $1 \%$ ammonium molybdate. 


\section{CHEMICAL SIGNALS FOR MOLECULAR REORGANIZATION}

Molecular reorganization within the lipid bilayers was monitored using the concentration dependent fluorescence properties of the pyrene probe. ${ }^{11}$ The pyrene moiety, which is situated in the hydrophobic region of the bilayer, produces a monomer emission $\left(\lambda_{\max }=376 \mathrm{~nm}\right)$ and excited state dimer, or excimer, emission $\left(\lambda_{\max }=470 \mathrm{~nm}\right)$ upon excitation at $346 \mathrm{~nm}$. The intensities of each emission are proportional to the population of the respective excited state species. Since the total pyrene concentration remains constant in the bilayer an increase in one excited state specie results in the equivalent loss of the other. The formation of excimer species is dependent upon the local concentration of the pyrene-labeled lipid. Aggregation of receptors in the membrane will result in an increased intensity of the excimer emission with concomitant decrease in monomer emission. Conversely, dispersion of the receptors will bring about the opposite fluorescence response.

In this section we will describe the fluorescence response of the functionalized bilayers to metal ions, proteins, and polypeptides. A molecular scheme is presented to describe the reorganizational process. Tailoring of the membrane's response to specific signals was achieved by altering the receptor's chemistry to target selected analytes.

\subsection{Fluorescence Spectral Changes Induced by Host-Guest Interactions}

The fluorescence spectra of the different bilayers containing $5 \%$ of a PS lipid in DSPC were very similar, all producing significantly larger excimer emission compared to the emission of the monomer. This was due to the phase separation phenomenon that occurs between the liquid phase PS lipids $\left(\mathrm{T}_{\mathrm{g}}<20^{\circ} \mathrm{C}\right)^{5}$ and the solid phase DSPC $\left(\mathrm{T}_{\mathrm{g}}=55^{\circ} \mathrm{C}\right)$. Crystalline domain formation of the DSPC forces the PS lipids into small patches between the crystal domains. The resultant high local concentration of the pyrene fluorophore gives rise to a strong excimer emission. Addition of certain divalent metal ions ${ }^{12}$, however, produces a shift in the intensities from the excimer and monomer emissions. Figure 5 shows a series of fluorescence spectra from 5\% PSIDA/DSPC bilayers in the presence of varying amounts of $\mathrm{MnCl}_{2}$. The ratio of the intensities of the excimer to monomer emission (E/M) are plot against the concentration of several metal ions producing the graph of Figure 6 for 5\% PSIDA/DSPC bilayers. Very high sensitivity and selectivity for $\mathrm{Cu}^{2+}$ was observed.

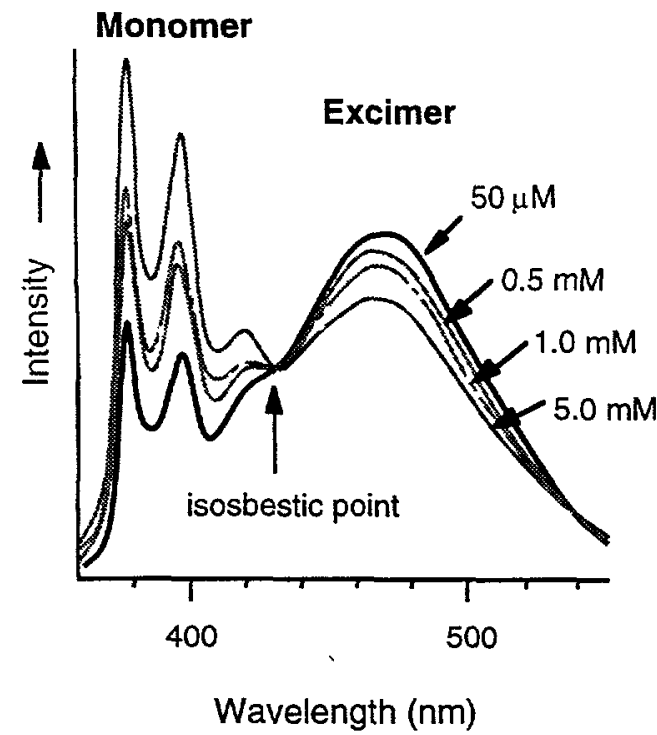

Figure 5. Fluorescence spectra of 5\% PSIDA/DSPC bilayers in aqueous MOPS buffer solution with varying amounts of $\mathrm{MnCl}_{2}$.

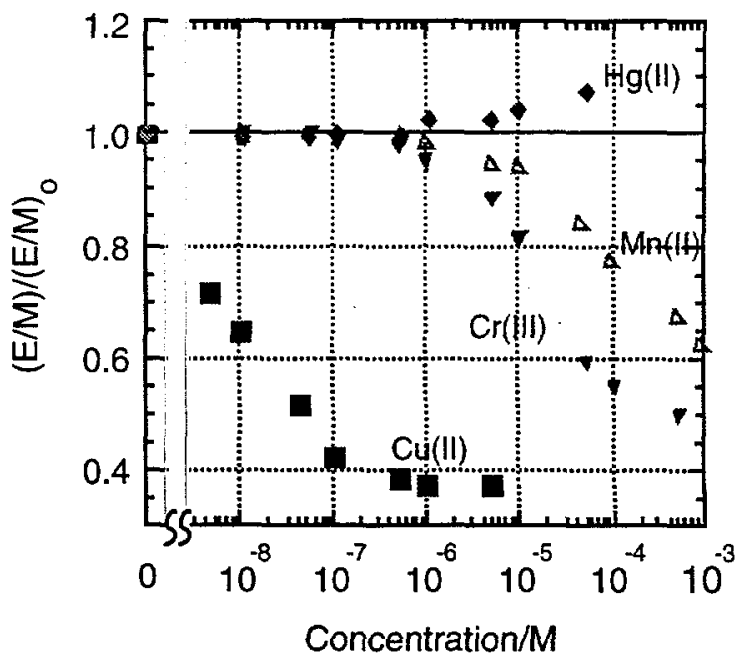

Figure 6. Graph of the normalized E/M response of $5 \%$ PSIDA/DSPC bilayers vs. concentration to several metal ions.

The changes in fluorescence induced by the presence of divalent metal ion has been determined to be due to a dispersion of the receptor lipids into the DSPC matrix initiated by selective metal ion binding by the receptor. Other possible causes, such as metal ion quenching of the pyrene fluorescence or non-specific metal ion interaction with the membrane producing changes in phase transition temperature, ${ }^{13}$ do not appear to influence the optical response. The presence of the isosbestic point in Figure 5 acknowledges the existence of only the two fluorescing species - the excited state monomer and excimer. If 
metal ion quenching occurs the overall fluorescence intensity would attenuate and an isosbestic point would not be observed. With regard to the specificity of the metal ion response, bilayers of 5\% PSOH/DSPC, which offer no metal ion receptor, were prepared and tested against a variety of different divalent and trivalent metal ions (e.g., $\mathrm{Cu}^{2+}, \mathrm{Ni}^{2+}, \mathrm{Hg}^{2+}, \mathrm{Zn}^{2+}, \mathrm{Cr}^{3+}, \mathrm{Mn}^{2+}$, $\left.\mathrm{Co}^{2+}, \mathrm{Ca}^{2+}\right)$. No response was observed for any of the metal ions at up to millimolar concentration. These results show that a specific metal ion interaction is required for a molecular reorganization and/or fluorescence response to occur.

The current understanding of the molecular reorganization process is illustrated in Figure 7 . The figure shows the outer leaflet of the lipid bilayer responding to metal ions. Initially, the receptors are aggregated as a result of phase separation of the DSPC matrix lipids from the PS lipids. Introduction of metal ion and capture by the receptors creates a positively charged complex. Ionic repulsion between receptors bound with metal ions causes these lipids to migrate into the DSPC matrix away from the unbound receptor aggregates. With increasing concentration of metal ion the receptor aggregate size decreases until no aggregates remain when all receptors are bound.

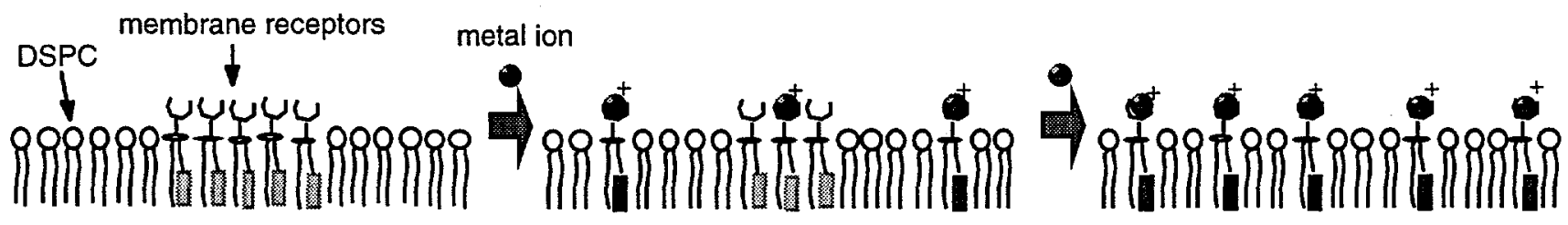

Figure 7. Illustration of metal ion induced molecular reorganization in the lipid membrane. The receptor lipids are initially aggregated (far left), but upon binding of metal ion and subsequent formation of charged headgroups the lipids repel each other and disperse into the matrix (middle). At high metal concentration all receptors are occupied and dispersion of receptors is at a maximum (far right).

This scenario requires that initially the receptor lipids have minimal repulsive forces so as not to diminish aggregate formation. For PSOH, PSDSDA, and PS18C6 lipids, ionic repulsion from charged headgroups will not occur in the absence of metal ion under the aqueous conditions of the present experiments. However, the PSIDA lipid can exist in four ionic states: positive at low $\mathrm{pH}$, zwitterionic and negative from low to neutral $\mathrm{pH}$, and doubly negative at high $\mathrm{pH}$. A pH profile with the $5 \%$ PSIDA/DSPC bilayers finds that between $\mathrm{pH} 7-8$ the $\mathrm{E} / \mathrm{M}$ ratio is largest indicating optimum aggregate formation of PSIDA (Figure 8). At pHs above 8 or below 7 the $\mathrm{E} / \mathrm{M}$ ratio decreases suggesting charge repulsion from the headgroups, that is cation formation below $\mathrm{pH} 7$ and anion formation above 8 . Between $\mathrm{pH} 7-8$ the zwitterionic form must exist allowing receptor aggregation. This is somewhat unexpected since in homogeneous solution the zwitterionic form of iminodiacetic acid is near $\mathrm{pH} 2 .^{14}$ However, changes in the $\mathrm{pK}_{\mathrm{a}}$ of functional groups have been known to be significantly different between derivatives existing in homogeneous solution to those on lipid membrane surfaces. ${ }^{15}$

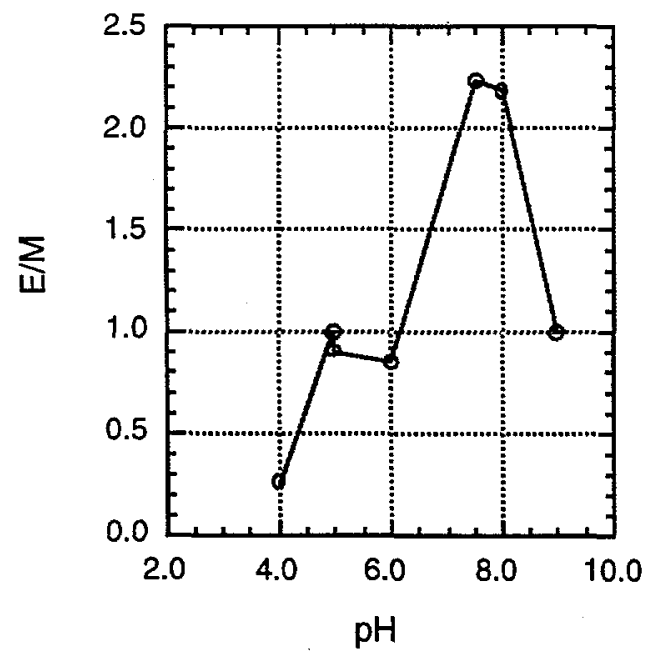

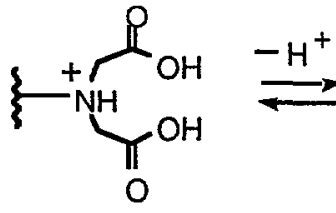

cationic<smiles>CC(C)(C)NC(=O)C(=O)O</smiles><smiles>C1=C[C+]2C=C1C2</smiles>

zwitterionic<smiles>CCN(CC(=O)OC)C(=O)OC</smiles>

anionic

Figure 8. Graph showing the effect of $\mathrm{pH}$ on the $\mathrm{E} / \mathrm{M}$ ratio of $5 \%$ PSIDADSPC lipid bilayers. On the right are three ionic states for iminodiacetic acid. 


\subsection{Molecular Reorganization Tailored to Specific Chemical Signals}

The binding of specific chemical signals to a membrane receptor induces a specific molecular reorganization in cellular membranes. Different chemical signals are used to trigger the reorganization of different sets of membrane components. Using our simple membrane receptors, we have found that the molecular reorganization process can be made specific to selected chemical signals via synthetic routes. The response curves of 5\% PSIDA/DSPC to heavy metal ions have already been presented in Figure 6. The response curves for 5\% PSDSDA/DSPC and 5\% PS18C6/DSPC are shown in Figures 9 and 10 , respectively. The PSIDA containing bilayer exhibited good selectivity for $\mathrm{Cu}^{2+}$. The PSDSDA containing bilayer, on the other hand, gives excellent selectivity for mercuric ion and the PS18C6 containing bilayer was selective for $\mathrm{Pb}^{2+}$. Dithioamides are efficient chelators of mercuric ion ${ }^{16}$ and crown ethers bind divalent lead ${ }^{17}$ with large association constants. Likewise, iminodiacetic acid, the headgroup of PSIDA, has the best association constant for cupric ion. ${ }^{14}$ Thus, the metal ion selectivity and membrane response is dictated by the headgroup chemistry.

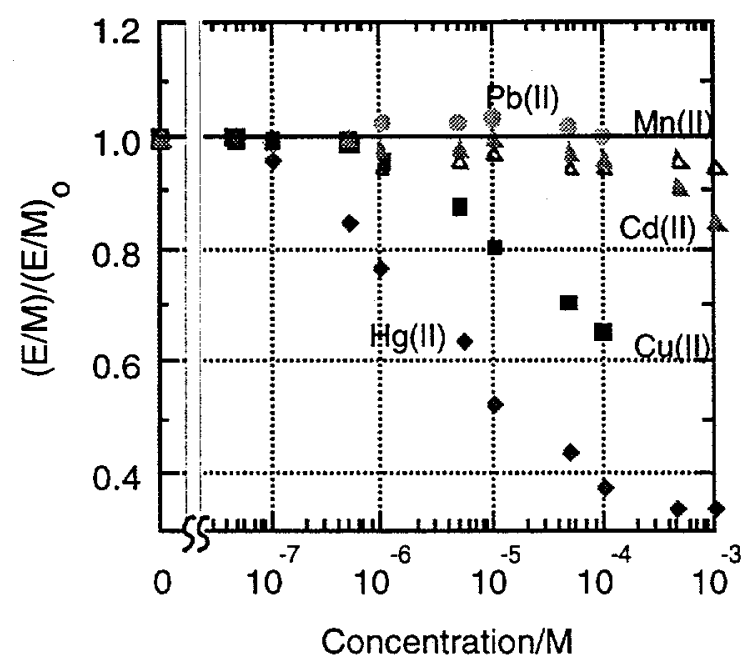

Figure 9. Metal ion response (E/M) of $5 \%$ PSDSDA/DSPC bilayers. These bilayers exhibited no response up to $10^{-4} \mathrm{M}$ concentrations for the following ions: $\mathrm{Pb}^{2+}, \mathrm{Ni}^{2+}, \mathrm{Co}^{2+}, \mathrm{Ca}^{2+}$.

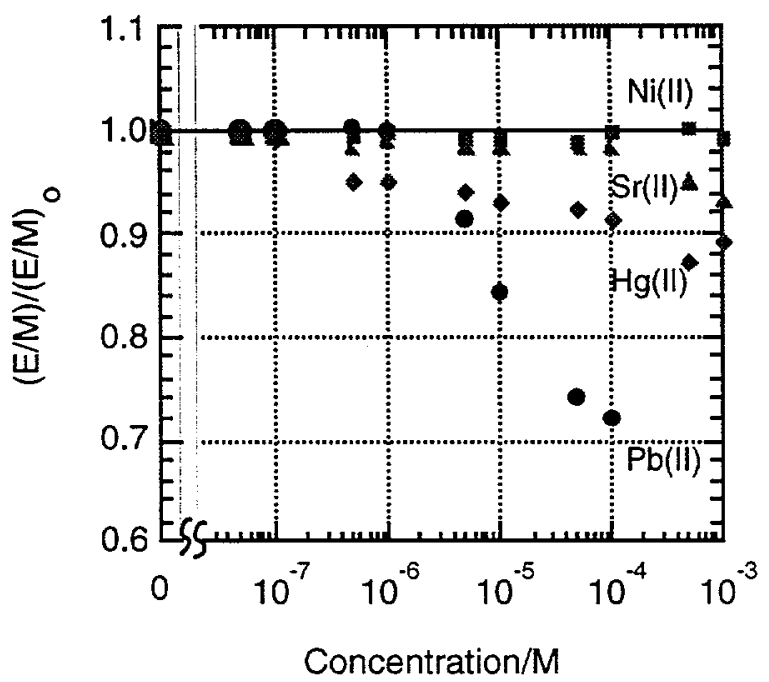

Figure 10. Metal ion response (E/M) of 5\% PS18C6/DSPC bilayers. These bilayers exhibited no response up to $10^{-4} \mathrm{M}$ concentrations for the following ions: $\mathrm{Cu}^{2+}, \mathrm{Ni}^{2+}, \mathrm{Ca}^{2+} \cdot \mathrm{Cr}^{3+}$. $\mathrm{Co}^{2+}, \mathrm{Cs}^{+}, \mathrm{Mn}^{2+}, \mathrm{Cd}^{2+}$.

The sensitivities and tunable selectivities of these optically responsive bilayers to metal ions make them attractive as sensor materials. For the 5\% PSIDA/DSPC, sensitivity limits for $\mathrm{Cu}^{2+}$ is at the sub-ppb level. Limits of sensitivities of $5 \%$ PSDSDA/DSPC for $\mathrm{Hg}^{2+}$ is in the ppb range, while that of $5 \% \mathrm{PS18C6/DSPC}$ for $\mathrm{Pb}^{2+}$ is at the sub-ppm level. For most metals, a linear E/M response was observed that could span up to three orders of magnitude of metal ion concentration. Additionally, since the E/M response is a ratio of the interconversion of two excited state species, the value is a more reliable and reproducible measurement compared to optical probes that rely on changes in intensity at a single wavelength. The spectral changes resulting from each metal ion response is the same for each bilayer, enabling the need for only one optical platform to monitor all sensor films. A coupled pattern recognition algorithm to a multi-probe sensor laced with different bilayer materials may provide for efficient characterization of mixed waste systems. The response is also completely reversible through removal of the bound metal ions with metal ion scavengers, such as EDTA.

These metal ion sensitive bilayers have also been used as selectively adsorbing surfaces for proteins and polypeptides. Previously, we have shown that monolayers of PSIDA chelated with $\mathrm{Cu}^{2+}$ exhibit strong adsorption for polyhistidine (poly(His)) and proteins tagged with poly(His). ${ }^{4,18,19}$ Fluorescence experiments provide evidence of the binding of these molecules to the film surface and the reorganization of pyrene lipids in response to the binding event. In this case, the binding interaction promotes the aggregation of initially dispersed receptors. In the presence of poly(His), monolayers of $5 \%$ PSIDA-Cu ${ }^{2+}$ in 95\% 1-stearyl-2-oleoyl-sn-glycero-phosphocholine (SOPC) produced large domains rich in the pyrene labeled lipid as indicated by the high intensity of excimer emission compared to the background observed with the fluorescence microscope. Bilayers of identical composition exhibited an increased $\mathrm{E} / \mathrm{M}$ ratio with the addition of submicromolar concentrations of poly(His). Multiple-point binding of poly(His) through the imidazoles to $\mathrm{Cu}^{2+}$-chelated- 
iminodiacetate brings about the aggregation of PSIDA lipids. Figure 11 illustrates this molecular reorganization. PSIDA$\mathrm{Cu}^{2+}$ is randomly oriented throughout the liquid phase SOPC matrix, but upon addition of poly(His), multiple imidazolePSIDA- $\mathrm{Cu}^{2+}$ binding interactions occur inducing lipid aggregation.

The ability to tailor molecular reorganization to specific chemical signals may someday allow us to organize multiple sites within a membrane with control over which site is reorganized and the precise order and timing of each reorganization. This added dimension of time in molecular organization might be a powerful asset in building complex structures.
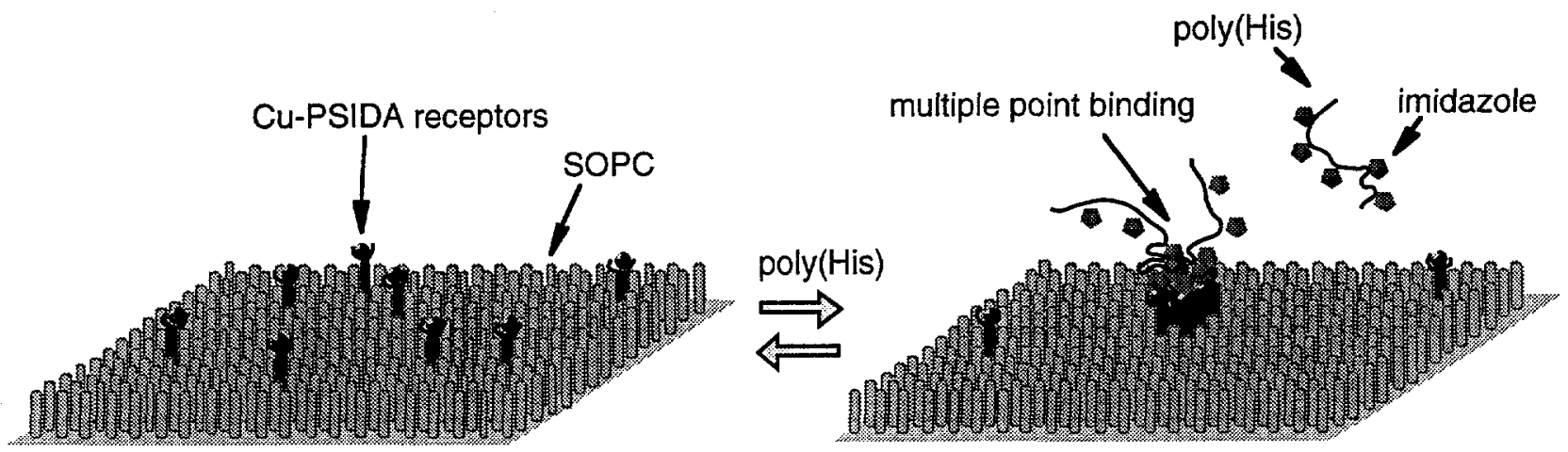

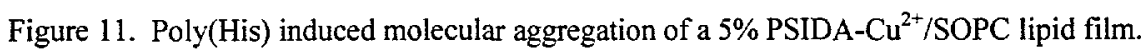

\section{SUMMARY}

Molecular reorganization via chemical signals is an extremely powerful tool used in cellular systems, for events such as adhesion, defense, division, food selection and uptake, etc. We have taken the first steps in the mimicry of these complex and well-orchestrated assemblies by reducing the system to simple chemical structures and physical phenomena. The membrane receptors were replaced with metal ion chelators, the chemical signals then became the metal ions, and the cellular membranes themselves were reduced to two component lipid bilayers. In our studies we have shown that rapid and reversible aggregation/dispersion of membrane components can be achieved using physical effects, such as phase separation of incompatible membrane components, ionic repulsion, and multiple-point binding interactions. The molecular reorganization can also be made specific to any chemical agent by altering the chemistry of the membrane receptor. A fluorescence technique used to probe the aggregational changes induced by metal ion binding also serves as an excellent signal transducer for the detection of metal ions. Materials for the sensitive and selective detection of $\mathrm{Cu}^{2+}, \mathrm{Hg}^{2+}$, and $\mathrm{Pb}^{2+}$ have been described. The next step in our path to understanding chemically driven molecular organization will involve the study of non-ionic molecular signals and signal binding effects on membrane topological structure.

\section{ACKNOWLEDGEMENTS}

Sandia is a multiprogram laboratory operated by Sandia Corporation, a Lockheed Martin Company, for the United States Department of Energy under Contract DE-AC04-94AL85000.

\section{REFERENCES}

1. B. Alberts, D. Bray, J. Lewis, M. Raff, K. Roberts, and J. D. Watson, Molecular Biology of The Cell, third ed. New York: Garland Publishing, 1994.

2. S. Miyamoto, S. K. Akiyama, and K. M. Yamada, "Synergistic Roles for Receptor Occupancy and Aggregation in Integrin Transmembrane Function," Science 267, pp. 883, 1995.

3. K. Eichmann, "Transmembrane Signaling of T Lymphocytes by Ligand-Induced Receptor Complex Assembly," Angew. Chem., Int. Ed. Engl. 32, pp. 54, 1993. 
4. K. Ng, D. W. Pack, D. Y. Sasaki, and F. H. Arnold, "Engineering Protein-Lipid Interactions: Targeting of HistidineTagged Proteins to Metal-Chelating Lipid Monolayers," Langmuir 11, pp. 4048, 1995.

5. D. Y. Sasaki, D. R. Shnek, D. W. Pack, and F. H. Arnold, "Metal-Induced Dispersion of Lipid Aggregates: A Simple, Selective, and Sensitivie Fluorescent Metal Ion Sensor," Angew. Chem., Int. Ed. Engl. 34, pp. 905, 1995.

6. D. Y. Sasaki and B. E. Padilla, "Dithioamide metal ion receptors on fluorescent lipid bilayers for the selective optical detection of mercuric ion," Chem. Comm., pp. 1581, 1998.

7. G. L. Gaines, Insoluble Monolayers at Liquid-Gas Interfaces. New York: John Wiley \& Sons, Inc., 1966.

8. A. Ulman, An Introduction to Ultrathin Organic Films. From Langmuir-Blodgett to Self-Assembly. San Diego: Academic Press, Inc., 1991.

9. R. R. C. New, Liposomes, 1 ed. New York: Oxford University Press, 1990.

10. D. D. Lasic, Liposomes: From Physics to Applications. Amsterdam: Elsevier Science, 1993.

11. H.-J. Galla and W. Hartmann, "Excimer-Forming Lipids in Membrane Research," Chem. Phys. Lipids 27, pp. 199, 1980.

12. All metal ions used in the studies reported herein are chloride salts

13. K. Jacobson and D. Papahadjopoulos, "Phase Transitions and Phase Separations in Phospholipid Membranes Induced by Changes in Temperature, pH, and Concentration of Bivalent Cations," Biochemistry 14, pp. 152, 1975.

14. A. E. Martell, P. M. Smith, Critical Stability Constants, Vol. 6, Plenum: New York, 1974.

15. N. A. Swairjo, B. A. Seaton, and M. F. Roberts, "Effect of vesicle composition and curvature on the dissociation of phosphatidic acid in small unilamellar vesicles - a 31P-NMR study," Biochim. Biophys. Acta 1191, pp. 354, 1994.

16. M. Lerchi, E. Bakker, B. Rusterholz, and W. Simon, "Lead-Selective Bulk Optodes Based on Neutral Ionophores with Subnanomolar Detection Limits," Anal. Chem. 64, pp. 1534, 1992.

17. C. Luca, H. A. Azab, and I. Tanase, "A Polarographic Study on Some Complexes of Lead(2+) and Cadmuim(2+) with Macrocyclic(crown ethers)," Anal. Chem. 18, pp. 449, 1985.

18. D. R. Shnek, D. W. Pack, D. Y. Sasaki, and F. H. Arnold, "Specific Protein Attachment to Artificial Membranes via Coordination to Lipid-Bound Copper," Langmuir 10, pp. 2382, 1994.

19. K. M. Maloney, D. R. Shnek, D. Y. Sasaki, and F. H. Arnold, "Fluorescence signaling of ligand binding and assembly in metal-chelating lipid membranes," Chem. Biol.3, pp. 185, 1996. 